\title{
Predicting Patterns of Beta Diversity in Terrestrial Vertebrates Using Physiographic Classifications in the Brazilian Cerrado
}

\author{
André Andrian Padial ${ }^{1,2, *}$, Luis Mauricio Bini ${ }^{1}$, José Alexandre Felizola Diniz-Filho ${ }^{1}$, \\ Nayara Pereira Resende de Souza ${ }^{1} \&$ Ludgero Cardoso Galli Vieira ${ }^{3}$
}

\author{
${ }^{1}$ Programa de Pós-graduação em Ecologia e Evolução, Departamento de Ecologia, ICB, \\ Universidade Federal de Goiás, Goiânia, GO, Brazil \\ ${ }^{2}$ Instituto Federal de Educação, Ciência e Tecnologia de Mato Grosso do Sul, \\ Campus Nova Andradina, Nova Andradina, MS, Brazil \\ ${ }^{3}$ Universidade de Brasília, Planaltina, DF, Brazil
}

\begin{abstract}
The effectiveness that environmental classifications have in representing terrestrial vertebrates inhabiting the Brazilian Cerrado was tested. Data on species composition of vertebrates were mapped on a cell's grid covering the Cerrado. These cells were classified according to environmental classification schemes and the classification strength (CS) of these schemes was tested. Classification schemes based on biological data were also generated. Moreover, the classification scheme generated using the biological data of one taxonomic group was used as criteria to calculate the CS values using the similarity matrix generated by a second taxonomic group, thus generating a cross-taxa CS. The environmental classification scheme at the fine spatial scale significantly represented the variability of terrestrial vertebrates found in the Cerrado; however, CS values were generally low. Cross-taxa CS values, although higher than those obtained by the environmental classifications, were also generally low and indicate that biological surrogates as predictors of overall biota have little effectiveness.
\end{abstract}

Key words: Biological Surrogates, Classification Strength, Environmental Surrogates, Physiographic Classification, Terrestrial Vertebrates.

\section{Introduction}

Conservation planning strategies are based recurrently on surrogates as a means to defy the Linnean (Raven \& Wilson 1992) and Wallacean shortfalls (Lomolino 2004). For instance, besides using specific taxonomic groups as surrogates for conservation targets (Rodrigues \& Brooks 2007) broad-scale biodiversity attributes have been used (e.g., land types, Harding \& Winterbourn 1997; Margules \& Pressey 2000). Several features (e.g., landform types, drainage basins, and dominant vegetation types) are used to generate groups of homogeneous areas that become the targets for conservation (Harding \& Winterbourn 1997; Silva et al. 2006). Heino \& Mykrä (2006) suggested that areas from different groups of a classification scheme should be preserved to maximize the conservation of the regional biodiversity.

\footnotetext{
${ }^{\star}$ Send correspondence to: Andre Andrian Padial Instituto Federal de Educação, Ciência e Tecnologia de Mato Grosso do Sul, Campus Nova Andradina, MS-473, Km 23, CEP 79750-000, Nova Andradina, MS, Brazil E-mail: aapadial@gmail.com
}

Silva et al. (2006) proposed a regionalization scheme for the Cerrado biome (Central Brazil). Five "landscape units" and 15 "ecological units" (nested within the landscape units) were delineated as important schemes in characterizing the spatial heterogeneity of the Cerrado biome. These schemes classify the biome in areas with similar general ecological conditions, and they can be used by managers as an objective criterion to delineate conservation areas (Silva et al. 2006). If the biota corresponds closely to the environmental classifications, each class of the physical classification scheme will encompass a portion of the overall variability of biodiversity (Heino \& Mykrä 2006). Nevertheless, the effectiveness of the landscape or ecological units proposed by Silva et al. (2006) as surrogates for conservation was not properly evaluated for any biological group.

We evaluated whether there was a correspondence between the classification schemes described above (hereafter referred to as a priori classifications) and the patterns of beta diversity generated by terrestrial vertebrates in the Cerrado biome (Brazil). We used data collected from 1199 species of mammals, birds, reptiles, and amphibians (see Diniz-Filho et al. 2008 for details about the biological 
data). We also evaluated whether there was a correspondence between the a priori classifications and the discrete units generated a posteriori using the biological data. The role of a given taxon as a biological surrogate of another taxon was evaluated with a cross-taxa analysis. For this analysis the classification generated by one taxonomic group was used to describe the patterns of beta diversity of a second taxonomic group.

\section{Methods}

Species' geographic ranges were mapped on a grid with a spatial resolution of $1^{\circ}$ that covered the area of the biome used to delineate the a priori classifications (see Silva et al. 2006 for detailed maps showing the $a$ priori classifications). With this grid we created a matrix of species presence-absence by cells ( $\mathrm{N}=133$ cells; see Diniz-Filho et al. 2008 for a partition of the Cerrado in 181 cells). The Jaccard similarity coefficient was used to calculate faunal similarity between cells in the biome for each taxonomic group.

We used the two classification schemes of the Cerrado biome (i.e., landscape units and ecological units) proposed by Silva et al. (2006). They used 41 environmental variables (see Silva et al. 2006 Table 1) in a cluster analysis to identify five landscape units that were characterized by dominant landform and vegetations types (see Figure 1 in Silva et al. 2006). A second classification scheme (with 15 classes) was formed by splitting the five landscape units into ecological units: five units of well-drained plains and plateaus dominated by savannas, three units of hilly terrains dominated by savannas, four units of plains dominated by deciduous and semi-deciduous forests, two units of plains with evergreen, semi-deciduous, and other forests and one unit of poorly drained lowlands dominated by seasonally flooding savannas (see Silva et al. 2006 for a detailed description of the different landscape and ecological units). Each cell in our grid was classified according to the categories provided by Silva et al. (2006) (Figure 1).

The grid system we used slightly differed from the area Silva et al. (2006) used to delineate the a priori classifications. As a consequence, three ecological units were excluded from our analysis: one ecological unit (3B) was not located in any of the cells we used, and two ecological units (2C and 3C) were only found in one cell. Thus, environmental classification at the fine spatial scale was comprised of 12 classes (Figure 1).

We calculated the classification strength (CS) of the a priori classifications for each taxonomic group (van Sickle \& Hughes 2000). The CS analysis measures the correspondence strength between a classification scheme and a matrix

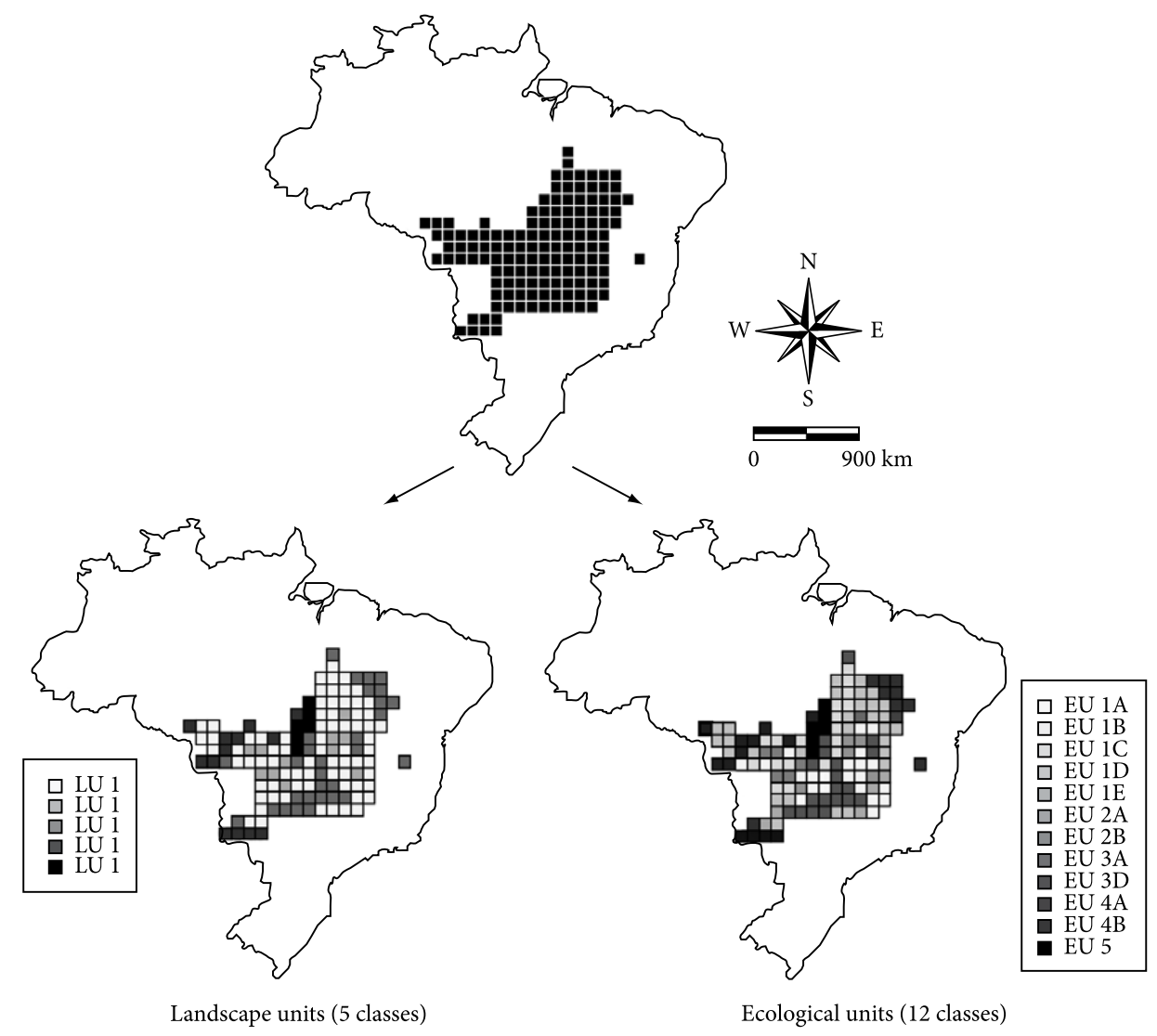

Figure 1. A map of Brazil showing the area of the Cerrado biome we analyzed (upper panel; divided into 133 grid cells of $1^{\circ} \times 1^{\circ}$ ) and the a priori classifications (lower panel; LU = Landscape units; EU = Ecological units) proposed by Silva et al. (2006). 
of biological data by calculating the difference between the average within-group similarity $(\mathrm{W})$ and the average between-group similarity (B). A large CS value (CS values vary from 0 to 1 , van Sickle \& Hughes 2000) indicates a high within-group and a low between-group similarity. In theory, this result indicates that the classes generated by a given classification scheme are valid to reproduce the main patterns of vertebrate beta-diversity. Significance levels of CS values were assessed by a Monte Carlo procedure with 10,000 permutations (Smith et al. 1990). The largest CS value found by comparing the two criteria indicates the classification scheme considered to be the best and most preferred scheme for conservation purposes.

We also used the $k$-means method to generate typologies of the Cerrado using the biological data (i.e., the a posteriori classifications). We reduced the dimensionality of the biological data with a non-metric multidimensional scaling (NMDS) technique to transform the binary data (presence/ absence) into a set of quantitative vectors more suitable for the Euclidian metrics used in the $k$-means method. We used the $k$-means clustering method to divide the landscape into the most appropriate and meaningful groups. As a method of determining when to stop adding groups to the analysis we used the following $R$ statistic (Hartigan 1975):

$\mathrm{R}=\left(\frac{\operatorname{tr}(\mathbf{W})(\mathrm{k})}{\operatorname{tr}(\mathbf{W})(\mathrm{k}+1)}-1\right)(\mathrm{n}-\mathrm{k}-1)$

where $\operatorname{tr}(\mathbf{W})$ is the trace of the dispersion matrix within groups, $n$ is the number of samples, and $k$ is the number of groups. This statistic was calculated for different values of $k$, and the results were compared to an $F$ distribution $(d f=p$; the number of variables, which was equal to two because we always used the first two axes of the NMDS for all biological groups). We calculated the CS values for the groups generated by the $k$-means method to compare the $a$ priori and a posteriori classifications. Finally, we used clusters generated by a given taxonomic group (in the surrogate role) as a classification criterion to calculate the CS values with the similarity matrix generated by a second taxonomic group (in the target role; see Pinto et al. 2007). Figure 2 summarizes our analytical approach.

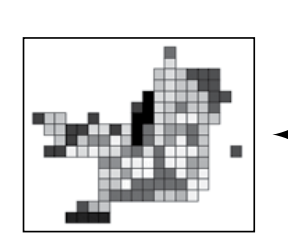

Ecological units (15 classess)

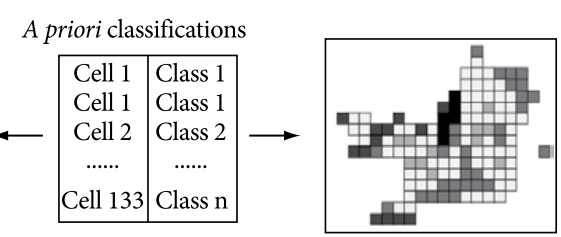

Lanscape units (5 classes)

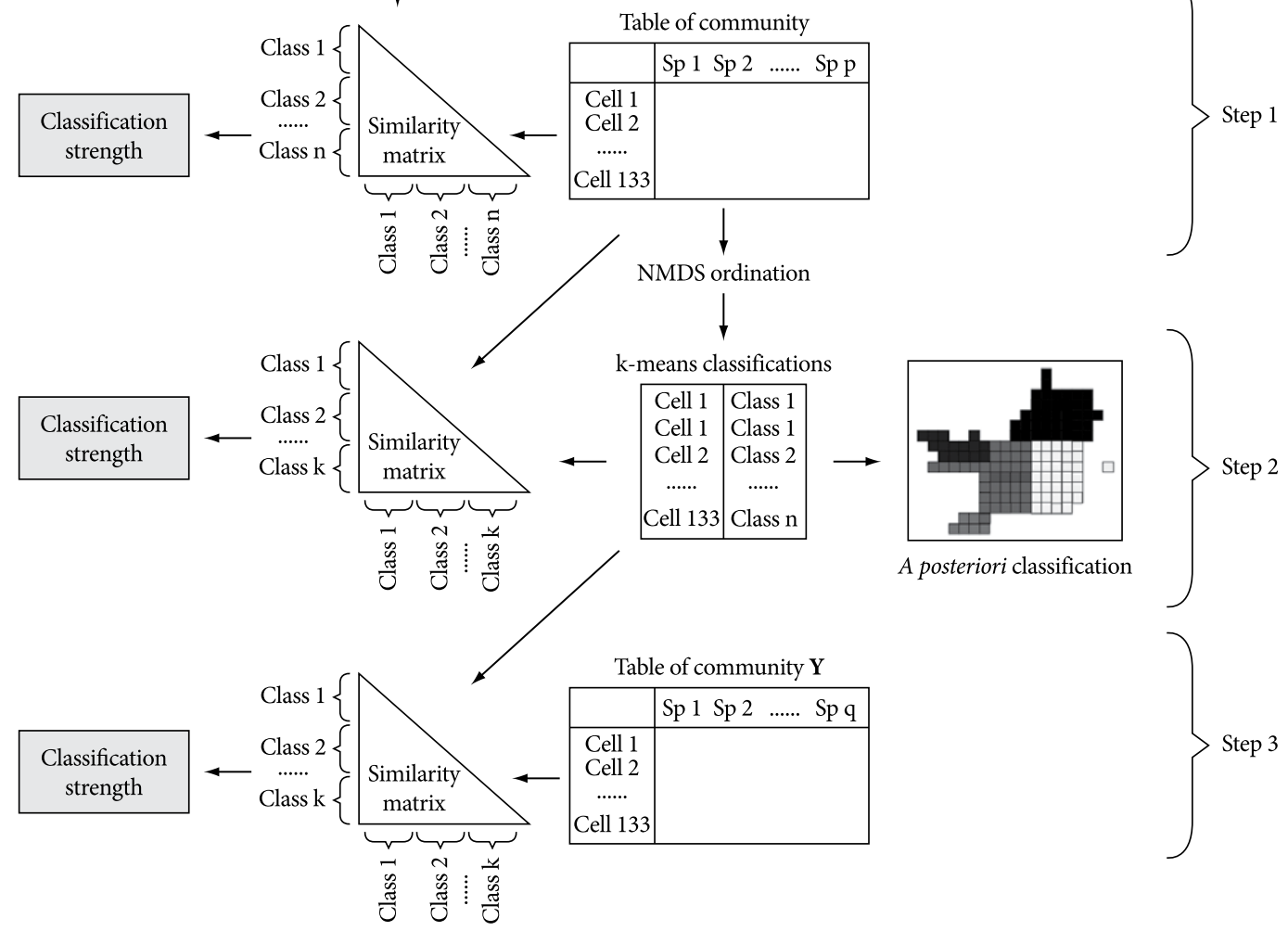

Figure 2. Our analytical approach. Step 1: Classification strength of the a priori classification schemes; Step 2: Classification strength of the a posteriori classification schemes; Step 3: Classification strength of the classification schemes based on a surrogate taxa. 


\section{Results}

CS values for the classification scheme based on the five landscape units were significant for bird and reptile species. The correspondences between the classifications based on the ecological units and the biological data were greater than those based on landscape units and significant for all vertebrate taxa (Table 1 ).

The number of classes generated by the $k$-means method varied for taxonomic groups (ranging from five for mammals to 12 for birds). As expected, the CS values of the a posteriori classifications were substantially higher than the CS values of the a priori classifications (Table 1); however, all CS were low (Table 1).

When the classification criterion generated by a given taxonomic group was used as surrogate for the other three taxonomic groups CS values, albeit low, the values were always significant and substantially higher than the values derived from the environmental data alone (Tables 1 and 2). The typology generated by birds received the highest CS values for the three other taxonomic groups.

Table 1. Classification strength for each a priori classification scheme. $\mathrm{B}=$ between group mean similarity; $\mathrm{W}=$ within-group mean similarity; CS = classification strength; $p=$ significance level based on 10,000 random permutations. The $a$ posteriori classifications were not tested because they were generated using the biological data, and thus they are a good and significant classification scheme.

\begin{tabular}{cccccccc}
\hline Classification scheme & Taxa & Number of classes & B & W & CS & p \\
\hline & Mammals & 5 & 0.604 & 0.608 & 0.003 & 0.356 \\
a priori & Birds & 5 & 0.648 & 0.669 & 0.021 & 0.014 \\
Landscape units & Reptiles & 5 & 0.415 & 0.432 & 0.017 & 0.025 \\
& Amphibians & 5 & 0.592 & 0.591 & -0.001 & 0.551 \\
\hline \multirow{2}{*}{ a priori } & Mammals & 12 & 0.600 & 0.661 & 0.061 & $<0.001$ \\
Ecological units & Birds & 12 & 0.652 & 0.709 & 0.058 & $<0.001$ \\
& Reptiles & 12 & 0.411 & 0.501 & 0.090 & $<0.001$ \\
& Amphibians & 12 & 0.585 & 0.647 & 0.063 & $<0.001$ \\
\hline \multirow{2}{*}{ a posteriori } & Mammals & 5 & 0.569 & 0.747 & 0.178 & - \\
& Birds & 12 & 0.635 & 0.851 & 0.215 & - \\
& Reptiles & 11 & 0.394 & 0.686 & 0.291 & - \\
\hline
\end{tabular}

Table 2. Results of the classification strength of a given taxonomic group (in the surrogate role; generated by a $k$-means method) in relation to other taxonomic groups (in the target role). $\mathrm{B}=$ between group mean similarity; $\mathrm{W}$ = within-group mean similarity; $\mathrm{CS}=$ classification strengths; $p=$ significance based on 10,000 random permutations

\begin{tabular}{cccccc}
\hline Target taxa & Surrogate classification & B & W & CS & p \\
\hline \multirow{3}{*}{ Mammals } & Birds (12 classes) & 0.583 & 0.828 & 0.245 & $<0.001$ \\
& Reptiles (11 classes) & 0.588 & 0.774 & 0.186 & $<0.001$ \\
& Amphibians (9 classes) & 0.580 & 0.808 & 0.228 & $<0.001$ \\
\hline \multirow{2}{*}{ Birds } & Mammals (5 classes) & 0.631 & 0.751 & 0.120 & $<0.001$ \\
& Reptiles (11 classes) & 0.644 & 0.760 & 0.115 & $<0.001$ \\
Reptiles & Amphibians (9 classes) & 0.637 & 0.799 & 0.162 & $<0.001$ \\
& Mammals (5 classes) & 0.390 & 0.544 & 0.154 & $<0.001$ \\
& Birds (12 classes) & 0.395 & 0.672 & 0.277 & $<0.001$ \\
Amphibians & Amphibians (9 classes) & 0.393 & 0.648 & 0.255 & $<0.001$ \\
& Mammals (5 classes) & 0.556 & 0.734 & 0.178 & $<0.001$ \\
& Birds (12 classes) & 0.571 & 0.790 & 0.218 & $<0.001$ \\
\hline \multirow{3}{*}{$*$} & Reptiles (11 classes) & 0.577 & 0.734 & 0.157 & $<0.001$ \\
\hline
\end{tabular}




\section{Discussion}

We found that the patterns of beta diversity in terrestrial vertebrates could not be predicted by the classifications based on the environmental and vegetation variability of the Brazilian Cerrado (Silva et al. 2006). Thus, these classification schemes cannot be considered reliable surrogates for biodiversity in conservation planning and cannot be used to establish a regionalization plan for monitoring terrestrial fauna in the Cerrado biome.

Weak CS values have been previously reported (e.g., Hawkins et al. 2000; Heino \& Mykrä 2006). In these studies, coarser environmental discontinuities, which determine the groups of a typology, may not have been predictive of beta diversity patterns (Brooks et al. 2004). The low correspondence found between the biological and environmental classifications may also relate to the means in which different taxonomic groups respond to local environmental factors (Heino et al. 2004). Correspondence between biota and climatic factors are evident and well-known at a global scale; however, local factors may be important for species within a biome. At a scale of landscape units (the largest spatial scale we used), low levels of beta diversity may have been found due to the ubiquity of common species of the biome (Higgins et al. 2005), which resulted in a high between-group similarity compared to the within-group similarity. In this case, the local environmental differences between cells found in each landscape unit may be ignored (Faith \& Walker 1996).

One could argue that the number of optimal a posteriori classes could differ from the number of optimal a priori classes and thus the CS values could not be compared. However, the "relative" CS may be calculated to circumvent such limitations (Snelder et al. 2005). For this calculation, the a posteriori classification scheme must be generated with the same number of classes as the a priori classification scheme. For instance, we can calculate the relative CS for mammals considering landscape units $(0.003 / 0.178)$ and for birds considering ecological units $(0.058 / 0.215$; note that the a priori and a posteriori classification schemes have the same number of classes in these cases; Table 1). The relative CS for mammals considering landscape units is $1.68 \%$, and the relative CS for birds considering ecological units is $26.97 \%$. These percentages indicate how good the a priori classification is in relation to the best classification possible (i.e., the a posteriori one, see also Snelder et al. 2005). It is clear that the a priori classifications have a low correspondence to the diversity of terrestrial vertebrates found in the Cerrado.

Congruent spatial patterns of different taxa would indicate the reliability of the use of biological surrogates of the overall biodiversity (Heino et al. 2005). This would be true if the classification scheme generated by a given taxonomic group (in the surrogate role) was valid for a second taxonomic group (in the target role). However, our results do not support the use of biological surrogates; the cross-taxa classifications strengths we found were also low. Effective surrogates cannot be considered as an alternative for a biodiversity analysis as they are difficult to find. Thus, if a taxonomic group or biodiversity pattern is considered important for conservation, the conservation targets and goals should be based directly on the available data for the specific group or pattern without trying to reach other indirect benefits (e.g., for other groups). Despite being necessary, surrogates for biodiversity conservation planning (Margules \& Pressey 2000) are generally unreliable.

Currently, most ecologists agree that using species data in systematic conservation planning is the best alternative method available in selecting a network of protected areas that reaches a given conservation goal at the lowest cost (Brooks et al. 2004). However, it is important to highlight that we are analyzing a biodiversity hotspot, the Cerrado, which has a high level of endemism and a high rate of habitat loss (Myers et al. 2000). We cannot wait for the proposition of conservation plans based solely on reliable data concerning species' occurrences (Pressey 2004). We can circumvent the paucity of species data using species distribution modeling, especially for rare species (Brooks et al. 2004; Guisan et al. 2006).

One can assume that considering environmental heterogeneity as an additional criterion in selecting priority areas for conservation would be a way to simultaneously encompass ecological processes and ecosystem services worthy of preservation (Higgins et al. 2004) and minimize biases caused by the paucity of biological data. Land types have been considered as entities relevant to conservation because they represent unique ecological processes and ecosystem services (Higgins et al. 2004; Molnar et al. 2004). Moreover, an area with idiosyncratic habitats and environmental conditions may have high conservation value because it may support endemic and/or rare species (Trakhtenbrot \& Kadmon 2006). However, assuming that the environmental variability properly represents the variability of the biota can lead to incorrect interpretations. At best, a multiple array of surrogates to circumvent idiosyncrasies of one approach may be a more comprehensive and stronger strategy for conservation planning purposes (Pressey 2004). Nevertheless, the use of either environmental or biological surrogates for conservation strategies does not exclude the urgent need for collecting baseline data on species distribution.

\section{Acknowledgements}

A.A. Padial and N.P.R. Souza received scholarships and acknowledge CAPES and CNPq for the financial support. L.M. Bini and J.A.F. Diniz-Filho also acknowledge CNPq for research grants. Financial support for this study came from a PRONEX program of CNPq/SECTEC-GO for establishing conservation priorities in Cerrado area. 


\section{References}

Brooks T, Fonseca GAB \& Rodrigues ASL, 2004. Species, data, and conservation planning. Conservation Biology, 18:1682-1688.

Diniz-Filho JAF et al., 2008. Spatial patterns of terrestrial vertebrate species richness in Brazilian Cerrado. Zoological Studies, 47:146-157.

Faith DP \& Walker PA, 1996. Environmental diversity: on the best-possible use of surrogate data for assessing the relative biodiversity of sets of areas. Biodiversity and Conservation, 5:399-415.

Guisan A et al., 2006. Using niche-based models to improve the sampling of rare species. Conservation Biology, 20:501-511.

Harding JS \& Winterbourn MJ, 1997. An ecoregion classification of the South Island, New Zealand. Journal of Environmental Management, 51:275-287.

Hartigan JA, 1975. Clustering Algorithms, New York: Wiley.

Hawkins CP et al., 2000. Evaluation of the use of landscape classifications for the prediction of freshwater biota: synthesis and recommendations. Journal of the North American Benthological Society, 19:541-556.

Heino J, Louhi P \& Muotka T, 2004. Identifying the scales of variability in stream macroinvertebrate abundance, functional composition and assemblage structure. Freshwater Biology, 49:1230-1239.

Heino J et al. 2005. Searching for biodiversity indicators in running waters: do bryophytes, macroinvertebrates, and fish show congruent diversity patterns? Biodiversity Conservation, 14:415-428.

Heino J \& Mykrä H, 2006. Assessing physical surrogates for biodiversity: do tributary and stream type classifications reflect macroinvertebrate assemblage diversity in running waters? Biological Conservation, 129:418-426.

Higgins JV et al., 2004. Beyond Noah: saving species is not enough. Conservation Biology, 18:1672-1673.

Higgins JV et al., 2005. A freshwater classification approach for biodiversity conservation planning. Conservation Biology, 19:432-445.

Lomolino MV, 2004. Conservation biogeography. In Lomolino MV \& Heaney LR (eds.), Frontiers of Biogeography: new directions in the geography of nature, Sunderland: Sinauer Associates, p. 293-296.

Margules CR \& Pressey RL, 2000. Systematic conservation planning. Nature, 405:243-253.

Molnar J, Marvier M \& Kareiva P, 2004. The sum is greater than the parts. Conservation Biology, 18:1670-1671.

Myers $\mathrm{N}$ et al. 2000. Biodiversity hotspots for conservation priorities. Nature, 403: 853-858.

Pinto MP et al., 2007. Biodiversity surrogate groups and conservation priority areas: birds of the Brazilian Cerrado. Diversity and Distributions, 14:78-86.

Pressey RL, 2004. Conservation planning and biodiversity: assembling the best data for the job. Conservation Biology, 18:1677-1681.

Raven PH \& Wilson EO, 1992. A fifty-year plan for biodiversity surveys. Science, 258:1099-1100.

Rodrigues ASL \& Brooks TM, 2007. Shortcuts for biodiversity conservation planning: the effectiveness of surrogates. Annual Review of Ecology, Evolution, and Systematics, 38:713-737.

Silva JF et al., 2006. Spatial heterogeneity, land use and conservation in the Cerrado region of Brazil. Journal of Biogeography, 33:536-548.

Smith EP, Pontasch KW \& Cairns J, 1990. Community similarity and the analysis of multi- species environmental data: a unified statistical approach. Water Research, 24:507-514.

Snelder TH, Biggs BJF \& Woods RA, 2005. Improved eco-hydrological classification of rivers. River Research and Applications, 21:609-628.

Trakhtenbrot A \& Kadmon R, 2006. Effectiveness of environmental cluster analysis in representing regional species diversity. Conservation Biology, 20:1087-1098.

van Sickle J \& Hughes RM, 2000. Classification strengths of ecoregions, catchments, and geographic clusters for aquatic vertebrates in Oregon. Journal of the North American Benthological Society, 19:370-384.

Received: April 2010

First Decision: June 2010 Accepted: August 2010 